Artículo de investigación E14A09. * Proyecto: “Criterios de medición y determinantes de revelación de activos intangibles para empresas cotizadas”. Universidad de Piura.

Recibido: 17.08.2018. * Aprobado versión final: 29.04.2020.

JEL: 41. Pp. 61-78. doi: 10.33571/teuken.v11n17a3

\title{
Determinantes de la revelación de activos intangibles para empresas cotizadas en Perú
}

\author{
Determinants of the intangible asset disclosure \\ for listed companies in Peru
}

\author{
Ivette Estefani Núñez Laguna - Julio César Hernández Pajares \\ PERÚ
}

\begin{abstract}
Resumen: Este artículo tiene como objetivo realizar un estudio descriptivo de la naturaleza de los activos intangibles y un análisis multivariante para determinar si los factores como sector empresarial, inversión en intangibles, tamaño de la empresa, endeudamiento y firma de auditoría influyen en el cumplimiento de la revelación de intangibles de acuerdo con la NIC 38. Se desarrolla un análisis de contenido de los reportes anuales de empresas cotizadas peruanas para los años 2016 y 2017 en torno a los criterios de medición y revelación de los activos intangibles. Los resultados indican que las empresas, en su mayoría, invierten en intangibles como licencias y softwares. Asimismo, el sector, la inversión en intangibles y la firma de auditoría resultan factores determinantes en el nivel de revelación.
\end{abstract}

Palabras claves: contabilidad; activos Intangibles; información financiera; niif; revelación financiera.

Abstract: The objective of this article is to carry out a descriptive study of the nature of intangible assets and a multivariate analysis to determine if factors such as business sector, investment in intangibles, company size, indebtedness and audit firm influence disclosure compliance. of intangibles in accordance with IAS 38. For this, a content analysis of the annual reports of Peruvian listed companies for the years 2016 and 2017 has been developed around the criteria for measurement and disclosure of intangible assets. The results indicate that the companies, for the most part, invest in intangibles such as licenses and software. Likewise, the sector, investment in intangibles and audit firm are determining factors in the level of disclosure.

Keywords: accounting; intangible assets; financial reporting; ifrs; financial disclosure.

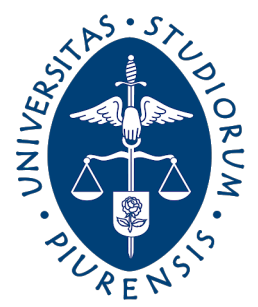

Ivette Estefani Núñez es Contadora Pública y Profesora asistente de cátedra de la Facultad de Ciencias Económicas y Empresariales, de la Universidad de Piura. Sus investigaciones se desarrollan en la línea de Información Financiera.

Contacto: ivette.nunez@udep.edu.pe

Julio César Hernández es Contador Público de la Pontificia Universidad Católica, Máster y Doctor en Contabilidad y Finanzas de la Universidad de Zaragoza. Profesor ordinario principal de la Facultad de Ciencias Económicas y Empresariales de la Universidad de Piura. Sus investigaciones se desarrollan en las líneas de Información Financiera e Información de Sostenibilidad.

Contacto: julio.hernandez@udep.edu.pe 


\title{
Determinantes da divulgação de ativos intangíveis para empresas listadas no Peru
}

\begin{abstract}
Resumo: O objetivo deste artigo é realizar um estudo descritivo da natureza dos ativos intangíveis e uma análise multivariada para determinar se fatores como setor de negócios, investimento em intangíveis, tamanho da empresa, endividamento e endividamento influenciam a conformidade da divulgação. de intangíveis de acordo com a IAS 38. Para isso, uma análise de conteúdo dos relatórios anuais das empresas listadas no Peru para os anos de 2016 e 2017 foi desenvolvida em torno dos critérios de mensuração e divulgação de ativos intangíveis. Os resultados indicam que as empresas, na maioria das vezes, investem em intangíveis, como licenças e software. Da mesma forma, o setor, o investimento em intangíveis e a empresa de auditoria são fatores determinantes no nível de divulgação.
\end{abstract}

Palavras-chave: contabilidade; ativos Intangíveis; informações financeiras; ifrs; divulgação financeira.

\section{Introducción}

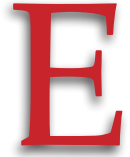

n cuanto al estudio de los criterios de reconocimiento y revelación de los activos intangibles en los estados financieros y otros reportes, las investigaciones señalan que su importancia radica en el problema que presentan en su medición confiable, capacidad de generar ingresos futuros y creación de valor en la empresa (Lev, 2004; Lev et al., 2005; Wyatt, 2008; Castilla-Polo, 2012; Ramírez y Gómez, 2015). A lo anterior, se suma la significación de la utilidad de la información financiera, según las Normas Internacionales de Información Financiera (NIIF), de proporcionar los criterios de reconocimiento y medición, así como de su presentación y revelación en los estados financieros y sus notas.

La aplicación completa de las NIIF vigentes tiene validez en Perú de acuerdo con la norma de la Comisión Nacional Supervisora de Empresas y Valores para empresas cotizadas desde los períodos comparados 20112012. Por último, la NIC 38, publicada por la International Accounting Standards Board (IASB) en 2001 como parte de las NIIF, tiene vigor a nivel internacional desde 2004 y establece los criterios de reconocimiento, medición y revelación de los activos intangibles. Habría que entender como tales a los activos identificables de carácter no monetario y sin apariencia física que cuentan con capacidad para generar beneficios económicos futuros.

Son relevantes las investigaciones sobre los factores como el tamaño, el sector, el nivel de endeudamiento y la firma de auditoría, y sobre el nivel de revelación de información financiera y, específicamente, de activos intangibles en países desarrollados (Oliveira et al., 2006; Chander \& Mehra, 
2011; Kang \& Gray, 2011; Devalle \& Rizzato, 2014; Azevedo et al., 2019) o en países de economías emergentes (Chander \& Mehra, 2011; Herrera, 2013; Ibadin \& Oladipupo, 2015; Salas et al., 2017; Agyei-Mensah, 2019;). Otros estudios se basan en la teoría de la agencia (Jensen \& Meckling, 1976; Wallace et al., 1994; Giner et al, 2003) y la teoría de costos del propietario (Hayes \& Lundholm, 1996; Miihkinen, 2012) para explicar las estrategias y riesgos de divulgación de la información financiera.

En nuestro contexto, existen estudios sobre activos intangibles como los de Valdivia (2012), Arrarte (2015) y Salas et al. (2017), quienes coinciden en señalar que se presentan limitaciones en la contabilidad financiera para reconocer y medir activos intangibles y su creación de valor. A pesar de ello, se encuentra un importante cumplimiento de aspectos de revelación de activos intangibles en las notas a los estados financieros de acuerdo con la NIC 38. Aparte de ello, estudios en Perú y otros países de la región latinoamericana han detectado convergencia de las prácticas contables relacionadas con los activos intangibles de acuerdo con la normativa del IASB o FASB (Kang \& Gray, 2011; Peruchena et al., 2015).

El objetivo de este artículo es realizar un estudio descriptivo de la naturaleza de los activos intangibles y un análisis multivariante para determinar si los factores como sector empresarial, inversión en intangibles, tamaño de la empresa, endeudamiento y firma de auditoría influyen en el cumplimiento de la revelación de intangibles de acuerdo con la NIC 38.

El artículo se compone, además de esta introducción, de una segunda parte que presenta antecedentes teóricos sobre la medición y revelación de activos intangibles y sus factores, así como el planteamiento de las hipótesis. La tercera parte explica el diseño de la investigación y la cuarta parte, el análisis de los resultados. Finalmente, se exponen las principales conclusiones.

\section{Marco teórico}

Las empresas que presentan activos intangibles, de acuerdo con la NIC 38, deben revelar información sobre los criterios de medición inicial y posterior bajo los modelos del costo o revaluación en la nota de políticas contables. Además, en las notas explicativas es necesario informar sobre la clasificación, amortización, conciliaciones de los valores al inicio y final del período y deterioro de los activos intangibles.

El análisis de los factores que influyen en el cumplimiento de revelación de los activos intangibles de la presente investigación ha considerado las teorías de la agencia y costos del propietario. Por un lado, los estudios que consideran la teoría de la agencia (Jensen \& Meckling, 1976; Leftwich 
et al., 1981) señalan que los directivos de las empresas más grandes y rentables tienen más incentivos de divulgar una mayor información para sus accionistas y acreedores con el fin de reducir sus costos de agencia y justificar su endeudamiento y resultados (Wallace et al., 1994; Ahmed \& Courtis, 1999; Giner et al., 2003). Por otro lado, la teoría de costos del propietario señala que la revelación de información tiene un costo para la empresa respecto a la preparación y publicación de información, así como un valor estratégico frente a sus competidores. Por tal motivo, los directivos optan por una menor revelación de información financiera para reducir dichos costos de propiedad (Darrough \& Stoughton, 1990; Hayes \& Lundholm, 1996; Birt et al., 2006; Miihkinen, 2012).

A partir de las teorías señaladas, consideramos las variables de tamaño y endeudamiento para medir los costos de agencia de las empresas (Jensen \& Meckling, 1976; Meek \& Gray, 1989), que varían, de acuerdo con el sector empresarial (Wallace et al., 1994) en la divulgación de la información financiera y presentan una importante influencia de las firmas de auditoría en dicha información (De Angelo, 1981).

\section{El sector}

Diversas investigaciones han destacado que el sector empresarial tiene incidencia en el nivel de información financiera revelada en los reportes anuales. La naturaleza de sus actividades y regulaciones específicas influyen en el nivel de divulgación de información de activos (Wallace et al., 1994; Cooke, 1992; Santos et al., 2014; Lang \& Stice-Lawrence, 2015) como el caso de empresas industriales, comunicación o servicios al consumidor (Meek et al., 1995; Gandía, 2003; Kang \& Gray, 2011). Esto implica, directamente, a las empresas con mayor inversión y uso de intangibles, como en las industriales y de servicios con uso intensivo de tecnología (Gandía, 2003; Oliveira et al., 2006; Kang \& Gray, 2011; Chander \& Mehra, 2011; Devalle et al., 2016). De acuerdo con lo señalado, planteamos las siguientes hipótesis:

H1: El sector empresarial tiene incidencia significativa en el cumplimiento de revelación de información de activos intangibles.

H2: La inversión en activos intangibles es determinante en el cumplimiento de revelación de información de activos intangibles.

\section{El tamaño}

La literatura académica considera que la divulgación de información financiera por las grandes empresas busca reducir los potenciales 
conflictos de intereses y, por lo tanto, los costos de agencia con los accionistas (Meek \& Gray, 1989; García y Sánchez, 2006). Se presume que estas empresas presentan un mayor número de usuarios potenciales que demandan la información que es atendida con una mayor divulgación en los reportes corporativos (Meek \& Gray, 1989; Lang \& Lundholm, 1993; Meek et al., 1995). En la misma línea, vale la pena tomar en cuenta que dichas empresas con mayor tamaño pueden tener un menor costo de preparación y mejor ventaja competitiva para presentar información a sus acreedores y obtener financiamiento del mercado público en mejores condiciones (Singhvi \& Desai, 1971; Meek et al., 1995; Giner et al., 2003).

La mayoría de estudios sobre divulgación de información de activos intangibles concluyen que el tamaño de la empresa medido por el valor de los activos resulta un factor determinante (Gandía, 2003; Oliveira et al., 2006; Herrera, 2013; Devalle \& Rizzato, 2014; Azevedo et al., 2019).

Por lo tanto, surge la siguiente hipótesis:

H3: El tamaño de las empresas es determinante en el cumplimiento de revelación de información de activos intangibles.

\section{El endeudamiento}

Teniendo en cuenta la teoría de la agencia, los directivos de las empresas con mayor nivel de endeudamiento divulgarán un mayor nivel de información financiera que muestre expectativas de resultados futuros de inversiones para favorecer el control a los accionistas (Jensen \& Meckling, 1976; Watts \& Zimmerman, 1986). En ese sentido, la información financiera ayudará a los acreedores e inversionistas a monitorear sus préstamos e inversiones (Wallace et al., 1994; Ahmed \& Courtis, 1999; Larrán y García, 2004; AgyeiMensah, 2019). En contraste, otros estudios no encontraron relación entre el nivel de endeudamiento y la divulgación de información financiera (Chow \& Wong-Boren, 1987; Meek et al., 1995; Gandía, 2003; García y Sánchez 2006). La falta de una mayor información para empresas con mayor endeudamiento se explica por los costos de propiedad y pérdidas en ventajas competitivas frente a su competencia (Macagnan, 2007).

Investigaciones analizadas señalan que el desarrollo y el tamaño del mercado de valores puede explicar la falta de relación del nivel de endeudamiento y el cumplimiento de revelación por las empresas, dado que la necesidad de revelar información será mayor por los requerimientos de los entes reguladores (García y Sánchez, 2006). En ese sentido, estudios sobre factores en la revelación de intangibles no encuentran una relación con el nivel de endeudamiento (Oliveira et al., 2006; Devalle \& Rizzato, 
2014) o señalan una relación negativa para el estudio de mercado de capitales emergentes, debido a que empresas con menor nivel de endeudamiento y mayor capital están interesados en informar sobre sus inversiones en intangibles (Kang \& Gray, 2011; Herrera, 2013).

Respecto a las evidencias anteriores, construimos la siguiente hipótesis:

H4: El nivel de endeudamiento de las empresas es determinante en el cumplimiento de información de activos intangibles.

\section{La firma de auditoría}

Las investigaciones revisadas encuentran que la naturaleza de la firma de auditoría influye en la divulgación de información financiera. Esto significa que, debido a su tamaño, las firmas buscan una mayor calidad para evitar riesgos del trabajo de auditoría, por lo que una adecuada revelación financiera asegurará dicha calidad (De Angelo, 1981; García y Sánchez, 2006). De esta manera, estas firmas de auditoría de mayor tamaño, denominadas "big four", influyen en los criterios de divulgación y en la presentación de información más detallada (Wallace \& Naser, 1995; Santos et al., 2014; Ibadin \& Oladipupo, 2015; Agyei-Mensah, 2019; Pocomucha y Hernández-Pajares, 2019).

Aunque los resultados no son concluyentes para la revelación de intangibles, en algunos casos, se ha encontrado que las firmas de auditoría más grandes son determinantes en el nivel de divulgación de información (Oliveira et al., 2006), mientras que en otros casos no es así (Devalle \& Rizzato, 2014).

Por estos antecedentes, se ha propuesto la siguiente hipótesis:

H5: El tamaño de la firma de auditoría es determinante en el cumplimiento de información de activos intangibles.

\section{Metodología}

Esta investigación es de naturaleza cuantitativa y longitudinal, con un alcance descriptivo y relacional de las variables sector, tamaño, inversión en intangibles, endeudamiento y firma de auditoría, con el cumplimiento de los criterios de revelación de la NIC 38 en las notas a los estados financieros. Por esta razón, se llevó a cabo un análisis de contenido de los reportes anuales de empresas cotizadas peruanas para los años 2016 y 2017. Para el análisis multivariante se utilizaron las herramientas estadísticas del SPSS 26.

La revisión del dictamen de auditoría y los estados financieros que permitieron obtener la información de las variables se obtuvieron de la base de datos de la Superintendencia del Mercado de Valores (SMV) en su página Web. 
El criterio de clasificación sectorial fue el de la Bolsa de Valores de Lima. Los sectores determinados han sido los siguientes: Agroindustria, Financiero, Comercial, Industrial, Minería, Seguros y Administradoras de Fondos de Pensiones, Servicios y Servicios Públicos (energía, agua y otros).

Para el análisis descriptivo y relacional se realizó una revisión exhaustiva del contenido de las notas a los estados financieros que comprende la nota de políticas contables y la de detalle de la partida intangibles para determinar su naturaleza y nivel de revelación (Valdivia, 2012; Peruchena et al., 2015; Salas et al, 2017).

Con respecto a la medición de la variable naturaleza del activo intangible, se revisó en la nota de detalle de la partida y se consideró, por ello, como variable categórica la clase de intangibles que la empresa revela.

En cuanto a la medición de la variable dependiente de nivel de cumplimiento de los criterios de revelación en notas a los estados financieros, primero se consideró una variable dicotómica, de 0 si no cumple y 1 si cumple cada uno de los siguientes criterios de revelación de la NIC 38 (Oliveira et al., 2006; Herrera, 2013; Devalle \& Rizzato, 2014):

- Criterios de medición inicial y posterior.

- Tipo de vida útil y métodos de amortización.

- Conciliación de los importes en iniciales y finales en el período del costo de los activos intangibles.

- Conciliación de los importes en iniciales y finales en el período de la amortización de los activos intangibles.

Posteriormente, se determinó un índice de medición de cumplimiento como el promedio de incidencias de criterios de revelación señalados y cumplidos (Bonsón y Escobar, 2004; Devalle \& Rizzato, 2014).

En relación con las variables independientes, la de tamaño de la empresa consideró el valor total de los activos y para la inversión en intangibles se aplicó la proporción respecto al total de activos (Cooke, 1992; Ahmed \& Courtis, 1999; Devalle \& Rizzato, 2014).

Para determinar la variable firma de auditoría se identificó la firma del dictamen que acompaña los estados financieros, y, para medir su tamaño, se clasificaron las 5 firmas según su ubicación en el ranking mundial por su volumen de ventas, que denominaremos "grandes" (PWC, E\&Y, Deloitte, KPMG y BDO), y las que no pertenecen al grupo anterior se agruparon como "otras". En función de ello, se midió la variable con 1 si pertenece al grupo de las "grandes" y 0 si pertenece al grupo de "otras" (Santos et al., 2014; Alanezi et al., 2015; Rouhou et al., 2015; Pocomucha y Hernández-Pajares, 2019). 
El nivel de endeudamiento se midió gracias a la aplicación del indicador de pasivo con respecto al patrimonio aplicado en estudios como factores de nivel de información (García y Zorio, 2002; Herrera, 2013; Lang \& Stice-Lawrence, 2015). La tabla 1 muestra las mediciones de las variables independientes que se consideran para la regresión lineal.

Tabla 1. Medición de variables independientes

\begin{tabular}{|l|l|}
\hline \multicolumn{1}{|c|}{ Variable } & \multicolumn{1}{c|}{ Medición } \\
\hline $\begin{array}{l}\text { Inversión en Intangibles } \\
\text { (Proporción) }\end{array}$ & Valor de Activos Intangibles/Nalor Total de Activos. \\
\hline $\begin{array}{l}\text { Tamaño de Empresa } \\
\text { Endeudamiento }\end{array}$ & Ln (Valor Total Activos). \\
\hline $\begin{array}{l}\text { Tamaño Firma } \\
\text { de Auditoría }\end{array}$ & $\begin{array}{l}\text { Valor Total de Pasivos/(Valor Total de Pasivo + Valor de Patrimonio). } \\
\text { de "otras". }\end{array}$ \\
\hline
\end{tabular}

Fuente: Elaboración propia.

Sobre las técnicas de análisis estadístico se aplicaron pruebas de ANOVA para medir el grado de incidencia del sector (variable categórica) en el nivel de cumplimiento de criterios de revelación (García y Moya, 2009; HernándezPajares 2018). Asimismo, se realizó un análisis de regresión lineal múltiple para evaluar la influencia significativa de las variables independientes en el nivel de cumplimiento de revelación (Oliveira et al., 2006; Kang \& Gray, 2011; Devalle \& Rizzato, 2014; Herrera y Macagnan, 2016).

\section{Muestra}

La información de las empresas se ha obtenido de la base de datos de la SMV. De la población total de 260 organizaciones que cotizaron en este mercado en los períodos 2016 y 2017, se excluyeron los fondos de inversiones, holding, organizaciones en liquidación y las empresas que no presentaron activos intangibles en su estado de situación financiera. La diferencia la constituyeron 127 empresas.

\section{Análisis de resultados}

\section{Análisis descriptivo}

Los gráficos 1 y 2 muestran las clases de activos intangibles revelados en notas a los estados financieros por las empresas analizadas para los años 2016 y 2017 teniendo en cuenta su sector empresarial, para ambos períodos, las licencias de software son el activo que más se revela por empresas financieras, industriales y de seguros y fondos de pensiones. 
Gráfico 1. Clase de intangibles revelados según sector para el período 2017

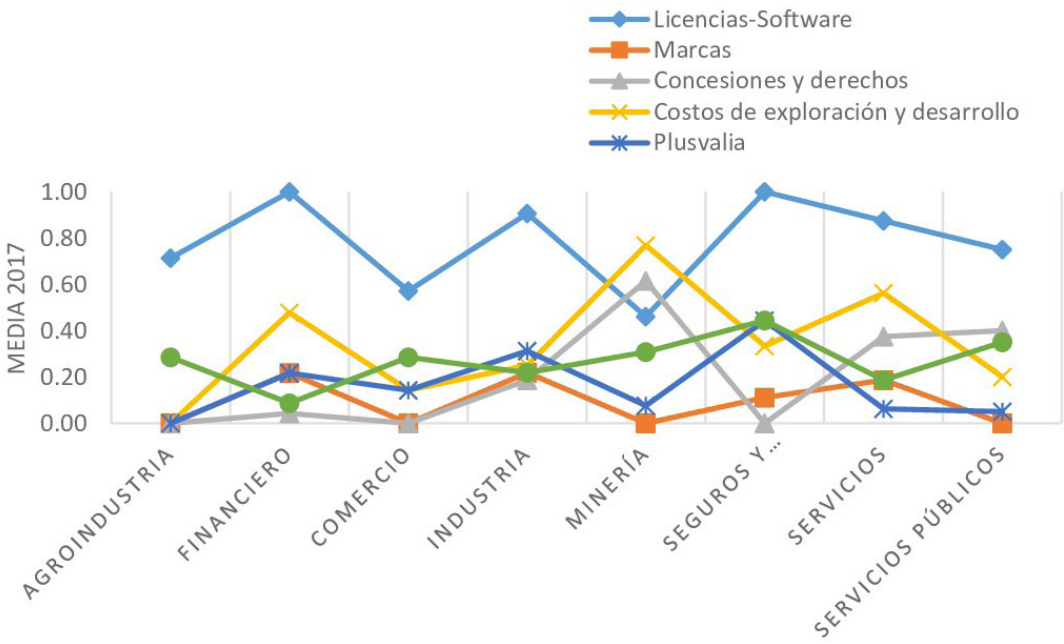

Fuente: Elaboración propia.

Gráfico 2. Clase de intangibles revelados según sector para el período 2016

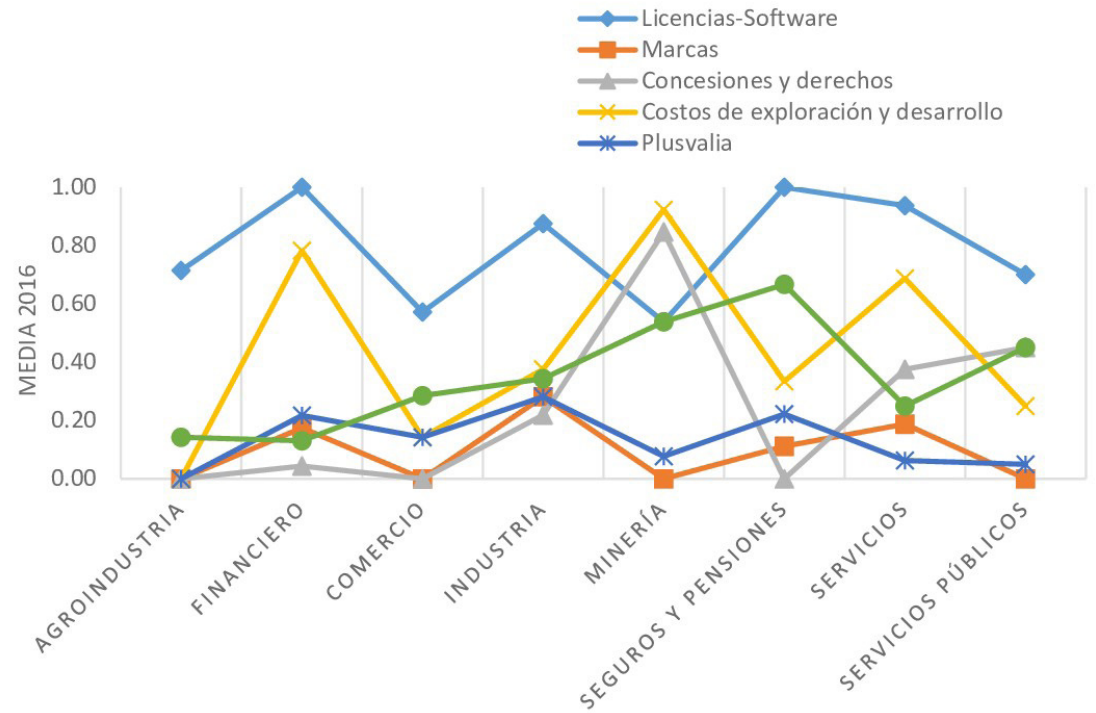

Fuente: Elaboración propia.

Las empresas financieras y de servicios divulgan, en mayor medida, costos de desarrollo de software y otros. Por otro lado, los costos de exploración y concesiones son lo más revelados por las empresas mineras. En tercer lugar, las marcas y plusvalía no son un intangible mayormente revelado, excepto para las empresas de seguros y fondos 
de pensiones que presentan plusvalías por adquisiciones y algunas industriales que invierten en marcas.

Respecto al nivel de cumplimiento de los criterios de revelación de los activos intangibles analizados en los estados de información financiera, se observó que no todas las empresas revelan la totalidad de la información exigida por la NIC 38. En algunos casos, no se revela información sobre la vida útil, método de amortización utilizado o la conciliación del importe en libros del costo y amortización de los activos intangibles. Esto se debe a que las empresas consideran la relevancia de revelar aspectos sobre los activos intangibles si la inversión en ellos es material o importante (Devalle \& Rizzato, 2014; Devalle et al., 2016).

El gráfico 3 muestra la media de cumplimiento de los criterios de revelación de intangibles por sector empresarial para ambos años 2016 y 2017 que resultan muy similares. Es evidente que no es uniforme para todos los sectores. Destaca el nivel de revelación en empresas con importantes inversiones en concesiones y costos de exploración (empresas mineras) y en software, licencias y costos de desarrollo (empresas de seguros y servicios). Por lo tanto, el tipo de industria influye en el nivel de inversión y revelación (Kang \& Gray, 2011; Chander y Mehra, 2011).

Gráfico 3. Media de índice de cumplimiento por sector de períodos 2016 y 201

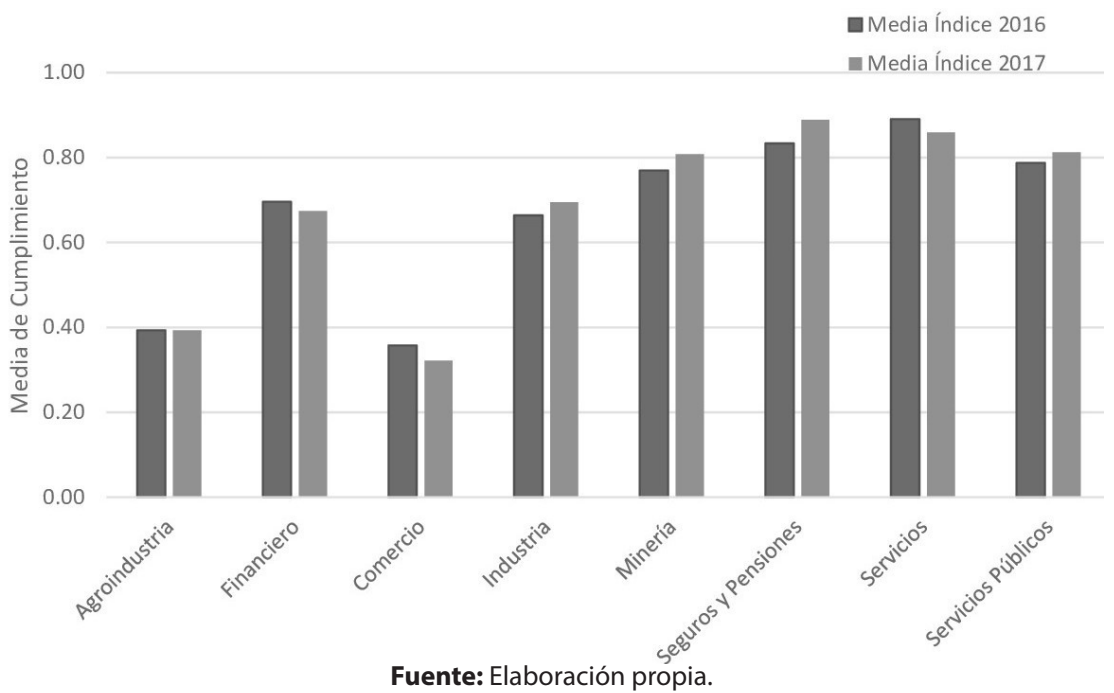

Finalmente, para analizar el efecto del tamaño de la firma de auditoría en el nivel de cumplimiento de los criterios de revelación se presenta el gráfico 4, en el cual se puede observar la media de cumplimiento de los criterios de revelación según las firmas de auditoría muy similar para los 
períodos 2016 y 2017. El nivel de revelación para las cinco mayores firmas de auditoría del grupo nombrado "grandes" es mayor que para las firmas del grupo denominado "otras" que, generalmente, corresponden a firmas locales (Santos et al., 2014; Agyei-Mensah, 2019).

Gráfico 4. Media de índice de cumplimiento por firma de auditoría. Períodos 2016 y 2017

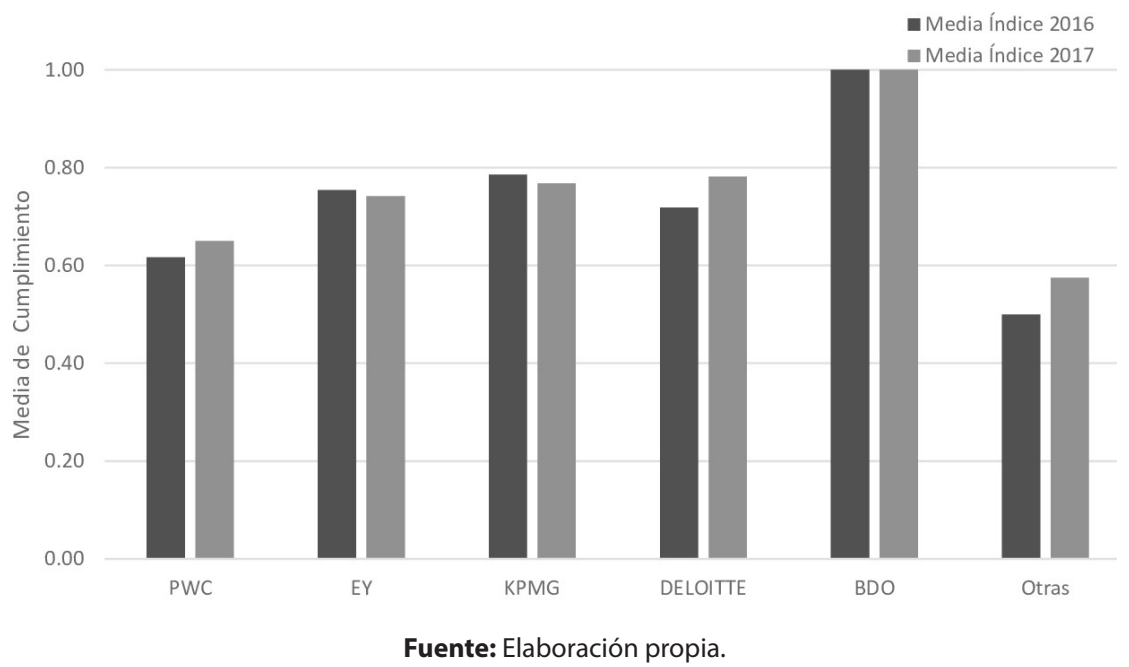

\section{Análisis multivariante}

Para medir la significatividad de la incidencia del sector empresarial en el nivel de cumplimiento de criterios de revelación de intangibles en notas a los estados financieros, realizamos una prueba ANOVA para los años 2017 y 2016. Los resultados de la tabla 2 señalan una incidencia significativa (Sig. 0,000<0,05 para el 2017 y Sig. 0,002<0,05 para el 2016), por lo que rechazamos la hipótesis nula y se acepta la hipótesis H1. Esto quiere decir que consideramos que el sector empresarial incide significativamente en el nivel de cumplimientos de los criterios de revelación. El nivel de revelación de intangibles no es el mismo según el tipo de sector: empresas que desarrollan actividades como minería, industria, de servicios y servicios públicos tiene una mayor inversión en intangibles en concesiones, software, costos de desarrollo que implican una mayor revelación (Gandía, 2003; Oliveira et al., 2006; Kang \& Gray, 2011; Chander \& Mehra, 2011).

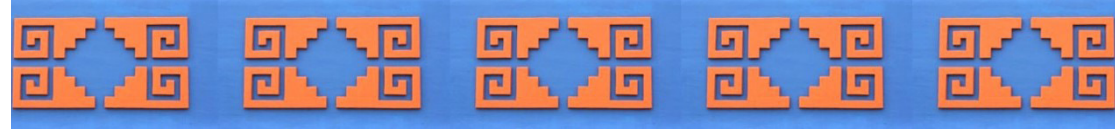


Tabla 2. Prueba ANOVA para nivel de cumplimiento de revelación según sector para los períodos 2017 y 2016

\begin{tabular}{|c|c|c|c|c|c|}
\hline Anova 2017 & $\begin{array}{c}\text { Suma de } \\
\text { cuadrados }\end{array}$ & gl & $\begin{array}{c}\text { Media } \\
\text { cuadrática }\end{array}$ & $\mathbf{F}$ & Sig. \\
\hline Entre grupos & 2,768 & 7 & ,395 & 4,963 & ,000 \\
\hline Dentro de grupos & 9,481 & 119 & ,080 & & \\
\hline Total & 12,249 & 126 & & & \\
\hline Anova 2016 & $\begin{array}{l}\text { Suma de } \\
\text { cuadrados }\end{array}$ & gl & $\begin{array}{c}\text { Media } \\
\text { cuadrática }\end{array}$ & $\mathbf{F}$ & Sig. \\
\hline Entre grupos & 81,929 & 4 & 20,482 & 4,660 & ,002 \\
\hline Dentro de grupos & 536,181 & 122 & 4,395 & & \\
\hline Total & 618,110 & 126 & & & \\
\hline
\end{tabular}

Fuente: Elaboración propia.

En cuanto al análisis de regresión lineal múltiple, se evaluó si la inversión en intangibles, tamaño de la empresa, nivel de endeudamiento y tamaño de la firma de auditoría son determinantes en el nivel de cumplimiento de criterios de revelación de intangibles. La tabla 3 muestra los estadísticos de contraste de regresión, con un nivel de significancia de 0,001 menor a 0,05 para ambos años, por lo que se pone de manifiesto que las variables independientes influyen de forma conjunta y lineal sobre la variable dependiente.

Tabla 3. Estadísticos regresión lineal (ANOVA) para nivel de revelación para los períodos 2017 y 2016

\begin{tabular}{|c|c|c|c|c|c|}
\hline Modelo 2017 & $\begin{array}{c}\text { Suma de } \\
\text { cuadrados }\end{array}$ & gl & $\begin{array}{c}\text { Media } \\
\text { cuadrática }\end{array}$ & $\mathbf{F}$ & Sig. \\
\hline Regresión & 1,699 & 4 &, 425 & 4,912 & ,001 \\
\hline Residuo & 10,550 & 122 & ,086 & & \\
\hline Total & 12,249 & 126 & & & \\
\hline Modelo 2016 & $\begin{array}{l}\text { Suma de } \\
\text { cuadrados }\end{array}$ & gl & $\begin{array}{c}\text { Media } \\
\text { cuadrática }\end{array}$ & $\mathbf{F}$ & Sig. \\
\hline Regresión & 1,708 & 4 &, 427 & 4,841 & ,001 \\
\hline Residuo & 10,762 & 122 & ,088 & & \\
\hline Total & 12,470 & 126 & & & \\
\hline
\end{tabular}

Fuente: Elaboración propia.

Las tablas 4 y 5 muestran los resultados de la regresión lineal para los períodos 2017 y 2016. Los resultados resaltan que el volumen de inversión en intangibles medido por su proporción con respecto al total de activos, se asocia positivamente y de forma significativa (Sig.0,000<0,05 para ambos años) con el nivel de cumplimiento de revelación de dichos activos. Con esto, confirmamos la hipótesis $\mathrm{H} 2$ y rechazamos la hipótesis nula de no asociación significativa: las empresas con mayor inversión en intangibles, y de uso intensivo relacionados con sus actividades, presentan una mayor información revelada en notas a los estados financieros (Gandía, 2003; Oliveira et al, 2006; Devalle \& Rizzato, 2014). 
No se ha observado que la variable de tamaño de las empresas medido por el valor de los activos resulte un factor determinante en la información revelada de intangibles. Por eso, rechazamos la hipótesis $\mathrm{H} 3$. Esto se traduce en que se evidenció que los directivos de las grandes empresas estudiadas no buscan una mayor información sobre activos intangibles para accionistas para reducir los costos de agencia ni se evita información que genere de costos de propiedad. En torno a esto, estudios como los de Kang \& Gray (2011) y Gandía (2003) no encuentran que el tamaño de las empresas se relaciona con el nivel de divulgación. A propósito, el presente estudio encuentra que es el tipo de industria y el nivel relevante de inversión en intangibles los que se relacionan con el nivel de revelación. Se ha hecho evidente, además, una relación negativa en cuanto a que se han descubierto grandes empresas que presentan un bajo nivel de revelación de activos intangibles como el caso de las comerciales y agroindustriales, que necesariamente no tienen una gran inversión en intangibles, como se observa en el gráfico 3.

Respecto al nivel de endeudamiento, aunque se presenta una relación positiva, no se encuentra que sea un factor determinante y significativo en el nivel de información de intangibles, por lo que rechazamos la hipótesis H4. Dado que el mercado de valores peruano no presenta una gran tamaño y desarrollo y el financiamiento de las empresas se desarrolla, principalmente, con bancos y no con emisión de acciones, puede no implicar una necesidad de cumplir mayores requerimientos de revelación con entes reguladores y accionistas (Depoers, 2000; García y Sánchez, 2006; Oliveira et al., 2006; Kang \& Gray, 2011). Al respecto, estudios como los de Depoers (2000), Devalle \& Rizzato (2014) y Azevedo et al. (2019) no encuentran que el nivel de endeudamiento sea un factor determinante.

La firma de auditoría resultó ser determinante en el nivel de divulgación de información sobre intangibles en las notas a los estados financieros. El tamaño de las firmas de auditoría se asocia significativa y positivamente con el nivel de revelación, por lo que no rechazamos la hipótesis 5 (H5) (Sig. 0,043<0,05 para el 2017 y Sig. 0,045<0,05 para el 2016). Las firmas de auditoría de mayor tamaño pertenecientes al grupo denominado "grandes" inciden en una mayor divulgación, probablemente, porque buscan reducir el riesgo de auditoría y cuidar su reputación. Por ello, incentivan, de alguna forma, a las empresas auditadas a que realicen un mayor cumplimiento normativo de revelación (García y Sánchez, 2006; Oliveira et al., 2006; Santos et al., 2014; Agyei-Mensah, 2019; Pocomucha y Hernández-Pajares, 2019). 
Tabla 4. Coeficientes de regresión lineal para el nivel de cumplimiento de revelación del período 2017

\begin{tabular}{|c|c|c|c|c|c|}
\hline \multirow[t]{2}{*}{ Variables } & \multicolumn{2}{|c|}{$\begin{array}{l}\text { Coeficientes no } \\
\text { estandarizados }\end{array}$} & \multirow{2}{*}{$\begin{array}{c}\begin{array}{c}\text { Coeficientes } \\
\text { estandarizados }\end{array} \\
\text { Beta }\end{array}$} & \multirow[t]{2}{*}{$\mathbf{t}$} & \multirow[t]{2}{*}{ Sig. } \\
\hline & B & Desv. Error & & & \\
\hline (Constante) &, 500 &, 214 & & 2.337 & 021 \\
\hline $\begin{array}{l}\text { Proporción } \\
\text { de Intangibles }\end{array}$ &, 531 & 145 & 311 & 3.675 &, $000^{*}$ \\
\hline Tamaño LnActivos &,- 005 & 017 &,- 028 &,- 294 & ,769 \\
\hline Endeudamiento & 131 & 119 & , 103 & 1.108 & 270 \\
\hline $\begin{array}{l}\text { Tamaño Firma } \\
\text { Auditoría }\end{array}$ & , 196 & 096 & 178 & 2.049 &, $043^{*}$ \\
\hline$R^{2}=0,139 \quad\left(^{*}\right)$ Sig. $<0,05$ & & & & & \\
\hline
\end{tabular}

Fuente: Elaboración propia.

Tabla 5. Coeficientes de regresión lineal para el nivel de cumplimiento de revelación del período 2016

\begin{tabular}{|c|c|c|c|c|c|}
\hline \multirow{2}{*}{ Variables } & \multicolumn{2}{|c|}{ Coeficientes no estandarizados } & \multirow{2}{*}{$\begin{array}{c}\begin{array}{c}\text { Coeficientes } \\
\text { estandarizados }\end{array} \\
\text { Beta }\end{array}$} & \multirow{2}{*}{$\mathbf{t}$} & \multirow{2}{*}{ Sig. } \\
\hline & B & Desv. Error & & & \\
\hline (Constante) &, 546 & ,219 & & 2,494 &, 014 \\
\hline Proporción de Intangibles &, 594 & , 162 & 310 & 3,655 &, $000^{*}$ \\
\hline Tamaño LnActivos &,- 006 & 017 &,- 034 &,- 365 & ,716 \\
\hline Endeudamiento & 039 & ,098 & ,036 & 400 & 690 \\
\hline Tamaño Firma Auditoría & ,203 & , 100 & , 174 & 2,027 &, $045^{*}$ \\
\hline$\left.{ }^{*}\right)$ Sig. $<0,05$ & & & & & \\
\hline
\end{tabular}

Fuente: Elaboración propia.

\section{Conclusiones}

Este estudio buscó contribuir con la investigación sobre la naturaleza de los activos intangibles y los factores de revelación de información en notas a los estados financieros de empresas cotizadas peruanas para los períodos 2016 y 2017.

Para ambos períodos, la actividad empresarial y el tipo de industria al que pertenecen las empresas, así como el volumen de inversiones en activos intangibles inciden en el nivel cumplimiento de información revelada. Se encontró que sectores empresariales específicos presentan inversiones significativas en activos intangibles. Por ejemplo, las empresas industriales exponen información de licencias, software y marcas; las industrias mineras revelan sobre costos de exploración y concesiones; las compañías de seguros y fondos de pensiones, servicios y servicios públicos divulgan sobre plusvalías, licencias, software y costos de desarrollo. Estos resultados son consistentes con otros estudios que señalan que el tipo de industria y el nivel de inversión en intangibles son factores que inciden en el nivel de revelación en notas a los estados financieros (Oliveira et al., 2006; Kang \& Gray, 2011; Chander \& Mehra, 2011). 
La teoría de la agencia no ha podido tomarse como un referente suficiente que explique el nivel de divulgación de información para las variables de tamaño y endeudamiento. En ese sentido, la realidad en Perú de un mercado de valores no muy desarrollado puede explicar que las empresas más grandes y con deuda privada no estén incentivadas para proporcionar una mayor información a accionistas y reducir los costos de agencia (Depoers, 2000; García y Sánchez, 2006; Kang \& Gray, 2011; Herrera, 2013).

El tamaño de la firma de auditoría resulta determinante en el nivel de revelación. Las cinco mayores firmas de auditoría en Perú clasificadas y denominadas "grandes" para esta investigación, que buscan cumplir con una alta calidad de trabajo de auditoría, pueden incentivar a las empresas que auditan al cumplimiento normativo en comparación con las firmas de auditoría locales de menor tamaño (DeAngelo 1981; Oliveira et al., 2006; Santos et al., 2014; Agyei-Mensah, 2019).

Esta investigación presentó algunas limitaciones. Se exhorta, en consecuencia, a que estudios futuros consideren un análisis comparativo con empresas de otros países de la región que posean naturaleza económica similar. Adicional a ello, para mejorar el coeficiente de determinación, se deben tener en cuenta otras variables independientes como rentabilidad, valor de capitalización bursátil o internacionalización de las empresas.

\section{Referencias bibliográficas}

1. Agyei-Mensah, B. K. (2019). IAS-38 disclosure compliance and corporate governance: evidence from an emerging market. Corporate Governance. The International Journal of Business in Society, 19, 419-437.

2. Ahmed, K. \& Courtis, J. K. (1999). Associations between corporate characteristics and disclosure levels in annual reports: a meta-analysis. The British Accounting Review, 31 (1), 35-61.

3. Alanezi, F. S.; Alfraih, M. M. \& Alshammari, S. S. (2015). Operating Segments (IFRS 8)-Required Disclosure and the Specific-Characteristics of Kuwaiti Listed Companies. International Business Research, 9 (1), 136-153. http://dx.doi.org/10.5539/ibr.v9n1p136

4. Arrarte, R. A. (2015). Contabilidad financiera y valuación de activos intangibles. Quipukamayoc, 23 (43), 9-20.

5. Azevedo, G.; Oliveira, J. \& Freitas, M. A. (2019). Compliance with intangible assets disclosure requirements: study of Portuguese non-financial companies. Contaduría y Administración, 64 (4), 1-34. http://dx.doi.org/10.22201/fca.24488410e.2018.1705

6. Bonsón, E. y Escobar, T. (2004). La difusión voluntaria de información financiera en WebWebt. Un análisis comparativo entre Estados Unidos, Europa del Este y la Unión Europea. Revista Española de Financiación y Contabilidad, 33 (123), 1063-1101. 
7. Birt, J. L., Bilson, C. M.; Smith, T. \& Whaley, R. E. (2006). Ownership, competition, and financial disclosure. Australian Journal of Management, 31 (2), 235-263. http://doi. org/10.1177/031289620603100204

8. Castilla-Polo, F. (2012). Divulgación voluntaria de intangibles y legitimación: La industria productora de aceite de oliva. Intangible capital, 8 (3), 564-600.

9. Chander, S. \& Mehra, V. (2011). A study on intangible assets disclosure: An evidence from Indian companies. Intangible Capital, 7 (1), 1-30. http://dx.doi.org/10.3926/ic.198

10. Chow, C. W. \& Wong-Boren, A. (1987). Voluntary financial disclosure by Mexican corporations. Accounting review, 62 (3), 533-541.

11. Cooke, T. E. (1992). The impact of size, stock market listing and industry type on disclosure in the annual reports of Japanese listed corporations. Accounting and business research, 22 (87), 229-237. https://doi.org/10.1080/00014788.1992.9729440

12. Darrough, M. N. \& Stoughton, N. M. (1990). Financial disclosure policy in an entry game. Journal of accounting and economics, 1990. 12 (1-3). 219-243.

13. Deangelo, L. (1981). Auditor size and audit quality. Journal of accounting and economics, 3 (3), 183-199.

14. Devalle, A. \& Rizzato, F. (2014). The determinants of the quality of mandatory disclosure of intangible assets under IFRS. GSTF Journal on Business Review (GBR), 3 (3), 23-29. https://doi.org/10.5176/2010-4804_3.3.320

15. Devalle, A.; Rizzato, F. \& Busso, D. (2016). Disclosure indexes and compliance with mandatory disclosure-The case of intangible assets in the Italian market. Advances in Accounting, 35, 8-25. https://doi.org/10.1016/j.adiac.2016.04.003

16. Depoers, F. (2000). A cost benefit study of voluntary disclosure: Some empirical evidence from French listed companies. European Accounting Review, 9 (2), 245-263. https://doi.org/10.1080/0963818005012989

17. Gandía, J. L. (2003). Intangibles Disclosure Information on Internet by Multinational Corporations. The International Journal of Digital Accounting Research, 3 (5), 61-99. https://doi.org/10.4192/1577-8517-v3_3

18. García, F. y Moya, I. (2009). Efecto de las NIIF en el valor bursátil de las empresas españolas. Investigaciones europeas de Dirección y Economía de la empresa, 15 (1), 61-79. https://doi.org/10.1016/S1135-2523(12)60078-1

19. García, E.y Sánchez, J. P. (2006). Un estudio meta-analítico de los factores determinantes de la revelación de información. Spanish Journal of Finance and Accounting, 35 (132), 761-788.

20. García, M. A. y Zorio, A. (2002). Características de las empresas europeas que aplican las normas del IASC: evidencia empírica de cara al debate regulador en la nueva fase de armonización contable. Spanish Journal of Finance and Accounting, 31 (111), 75110. https://doi.org/10.1080/02102412.2002.10779445

21. Giner, B.; Cervera, N.; Ruiz, A. y Arce, M. (2003). Incentivos para la divulgación voluntaria de información: evidencia empírica sobre la información segmentada. Revista europea de dirección y economía de la empresa, 12 (4), 69-85.

22. Ibadin, P. \& Oladipupo, O. A. (2015). Determinants of intangible assets disclosure in quoted companies in Nigeria. Asian Journal of Accounting \& Governance, 6, 13-25. http://dx.doi.org/10.17576/ajag-2015-6-12171

23. Hayes, R. M. \& Lundholm, R. (1996). Segment reporting to the capital market in the presence of a competitor. Journal of Accounting Research, 34 (2), 261-279. 
24. Hernández-Pajares, J. (2018). Influencia de la naturaleza internacional de empresas peruanas en su información de sostenibilidad. Revista de Comunicación, 17 (1), 74-92.

25. Herrera, E. E. (2013). Factores que explican la extensión de revelación de activos intangibles de los bancos que cotizan en la Bolsa de Valores de Panamá. Contaduría y Administración, 58 (3), 173-202. https://doi.org/10.1016/S0186-1042(13)71226-9

26. Herrera, E. E. y Macagnan, C. B. (2016). Revelación de informaciones sobre capital estructural organizativo de los bancos en Brasil y España. Contaduría y Administración, 61 (1), 4-25. https://doi.org/10.1016/j.cya.2015.09.007

27. Jensen, M. C. \& Meckling, W. H. (1976). Theory of the firm: Managerial behavior, agency costs and ownership structure. Journal of financial economics, 3 (4), 305-360.

28. Kang, H. H. \& Gray, S. J. (2011). Reporting intangible assets: Voluntary disclosure practices of top emerging market companies. The international journal of accounting, 46 (4), 402-423. https://doi.org/10.1016/j.intacc.2011.09.007

29. Lang, M. \& Lundholm, R. (1993). Cross-sectional determinants of analyst ratings of corporate disclosures. Journal of accounting research, 31 (2), 246-271.

30. Lang, M. \& Stice-Lawrence, L. (2015). Textual analysis and international financial reporting: Large sample evidence. Journal of Accounting and Economics, 60 (2-3), 110-135. http://dx.doi.org/10.2139/ssrn.2407572

31. Larrán, M. y García, E. (2004). La relevancia de la información no-financiera en la estrategia empresarial de divulgación unitaria: percepciones empresa-analista sobre su utilidad, Revista Valenciana de Economía y Hacienda, 12, 127-14.

32. Leftwich, R. W.; Watts, R. L. y Zimmerman, J. L. (1981). Voluntary corporate disclosure: The case of interim reporting. Journal of accounting research, 19, 50-77.

33. Lev, B. (2004). Intangibles en la encrucijada. Revista de contabilidad y dirección, 1, 15-29.

34. Lev, B.; Cañibano, L. \& Marr, B. (2005). An accounting perspective on intellectual capital (42-55). En B. Marr (Ed.), Perspectives on intellectual capital. https://doi.org/10.1016/ B978-0-7506-7799-8.50008-5

35. Macagnan, B. (2007). Condicionantes e implicación de revelar activos intangibles. (Tesis doctoral), Universitat Autónoma de Barcelona, España.

36. Meek, G. K. \& Gray, S. J. (1989). Globalization of stock markets and foreign listing requirements: Voluntary disclosures by continental European companies listed on the London Stock Exchange. Journal of international business studies, 20 (2), 315-336.

37. Meek, G. K.; Roberts, C. B.; Gray, S. J. (1995). Factors influencing voluntary annual report disclosures by US, UK and continental European multinational corporations. Journal of international business studies, 26 (3), 555-572.

38. Miihkinen, A. (2012). What drives quality of firm risk disclosure? The impact of a national disclosure standard and reporting incentives under IFRS. The International Journal of Accounting, 47 (4), 437-468. https://doi.org/10.1016/j.intacc.2012.10.005

39. Oliveira, L.; Rodrigues, L. \& Craig, R. (2006). Firm-specific determinants of intangibles reporting: evidence from the Portuguese stock market. Journal of Human Resource Costing \& Accounting, 10 (1), 11-33. https://doi.org/10.1108/14013380610672657

40. Peruchena, J. L.; Kronbauer, C. A.; Ott, E. y Moreno, J. (2015). Medición y Evidenciación de los Activos Fijos e Intangible en empresas Industriales del Mercosur y la Comunidad Andina. Revista Contabilidade e Controladoria, 7 (1), 111-132. http:// dx.doi.org/10.5380/rcc.v7i1.37569 
41. Pocomucha, K. y Hernández-Pajares, J. (2019). Influencia de la firma de auditoría y desempeño de empresas cotizadas en la extensión de revelación de información de los reportes anuales. Revista Contabilidad \& Sistemas, 16, 46-54.

42. Ramírez, Z. y Gómez, A.M. (2015). Grado de conocimiento del adjetivo "intangibles", y su relación con la identificación, reconocimiento, medición, valoración y revelación de" intangibles" en la información contable de las empresas en Popayán. Un análisis descriptivo y econométrico. Cuadernos de Contabilidad, 16 (40), 111-150. https://doi. org/10.11144/Javeriana.cc16-40.gcai

43. Rouhou, N. C.; Mrad-Douagi, W. B. Y Hussainey, K. (2015). The effect of IFRS enforcement factors on analysts' earnings forecasts accuracy. Corporate Ownership and Control, 13 (1-2), 266-282.

44. Salas, J.; Vilchez, P. y Curvelo, J. O. (2017). Claridad y calidad de las notas explicativas en estados financieros bajo IFRS. Un estudio de brechas de Expectativas Gaps para normas contables (NIC 2, NIC 16, NIC 37 y NIC 38). Quipukamayoc, 25 (49), 27-40. https://doi.org/10.15381/quipu.v25i49.14277

45. Santos, E.; Ponte, V. \& Mapurunga, P. (2014). Mandatory IFRS adoption in Brazil (2010): Index of compliance with disclosure requirements and some explanatory factors of firms reporting. Revista Contabilidade \& Finanças, 25 (65), 161-176. https://doi. org/10.1590/S1519-70772014000200006

46. Singhvi, S. \& Desai, H. (1971). An empirical analysis of the quality of corporate financial disclosure. The Accounting Review, 46 (1), 129-138.

47. Valdivia, R.A. (2012). MÁS ALLÁ... DE LA NIC-38 [Una mirada al futuro de los Activos Intangibles]. Quipukamayoc, 20 (38), 92-101. https://doi.org/10.15381/quipu.v20i38.4435

48. Wallace, R. S.; Naser, K. \& Mora, A. (1994). The relationship between the comprehensiveness of corporate annual reports and firm characteristics in Spain. Accounting and business research, 25 (97), 41-53.

49. Wallace, R.S. \& Naser, K. (1995). Firm-specific determinants of the comprehensiveness of mandatory disclosure in the corporate annual reports of firms listed on the stock exchange of Hong Kong. Journal of Accounting and Public Policy, 14 (4), 311-368.

50. Watts, R. L. \& Zimmerman, J. L. (1986). Positive Accounting Theory. Englewood Cliffs, Prentice-Hall.

51. Wyatt, A. (2008). What financial and non-financial information on intangibles is valuerelevant? A review of the evidence. Accounting and business Research, 38 (3), 217256. https://doi.org/10.1080/00014788.2008.9663336

Para citar este artículo:
Núñez, I. E. y Hernández, J. C. (2020). Determinantes de la revelación de activos intangibles para empresas cotizadas en Perú. Teuken Bidikay, 11 (17), 61-78. doi: 10.33571/teuken.v11n17a3

Ge: AMV. 